\title{
BMJ Open Protocol for active safety monitoring of a cohort of patients using a dolutegravir- based antiretroviral regimen in Mozambique
}

\author{
Merana Mussá, ${ }^{1}$ Irénio Gaspar, ${ }^{2}$ Luisa Namburete, ${ }^{1}$ Tânia Vuyeya Sitoie, ${ }^{1}$ \\ Aleny Couto, ${ }^{2}$ Jamal Mario Paulino, ${ }^{1}$ Abrao Lemos, ${ }^{1}$ Stefia Vilanculos, ${ }^{1}$ \\ Comfort Kunak Ogar (10 , ${ }^{3}$ Denylson Namburete, ${ }^{4}$ Eunice Dias Seni, ${ }^{4}$ \\ Tamara Hafner, ${ }^{5}$ Andy Stergachis ${ }^{6,7}$
}

To cite: Mussá M, Gaspar I, Namburete L, et al. Protocol for active safety monitoring of a cohort of patients using a dolutegravir-based antiretroviral regimen in Mozambique. BMJ Open 2021;11:e050671. doi:10.1136/ bmjopen-2021-050671

- Prepublication history and additional supplemental material for this paper are available online. To view these files, please visit the journal online (http://dx.doi.org/10.1136/ bmjopen-2021-050671).

Received 05 March 2021 Accepted 25 August 2021

Check for updates

(C) Author(s) (or their employer(s)) 2021. Re-use permitted under CC BY-NC. No commercial re-use. See rights and permissions. Published by BMJ.

For numbered affiliations see end of article.

Correspondence to Ms Comfort Kunak Ogar; kunacom@yahoo.com

\section{ABSTRACT}

Introduction Dolutegravir-based antiretroviral therapy (ART) is increasingly being used as the preferred firstline regimen for the treatment of HIV in low-income and middle-income countries. The National Program for the Control of STI/HIV/AIDS in Mozambique has planned a phased introduction of the tenofovir/lamivudine/ dolutegravir (TLD) regimen. In 2019, concerns about a potential safety signal identified with dolutegravir identified in the results of the Tsepamo study, conducted in Botswana, led the National Directorate of Pharmacy and the National Program for the Control of ST//HIV/AIDS to establish an active pharmacovigilance surveillance system among newly placed patients on a TLD regimen. This activity aims to establish an active pharmacovigilance system to monitor adverse events in patients on a TLD regimen to support the effectiveness of Mozambique's public health programmes in improving the process of care and treatment outcomes for people with HIV/AIDS.

Methods and analysis This is a prospective, noninterventional, descriptive cohort study to monitor HIV patients managed with TLD at 10 sentinel health centres in Mozambique. The cohort consists of HIV-infected patients commencing treatment with TLD, either as treatment naïve patients or switched from other ART regimens. Patients have monthly routine follow-up visits for the first 3 months after starting HIV treatment with TLD, and subsequently every 3 months for a total period of 1 year. Patients are monitored to identify possible adverse events during the follow-up period. The intended size of the cohort is 3000 patients.

Ethics and dissemination Ethical approval was obtained from the National Commission on Bioethics in Health in Mozambique. Written informed consent is obtained from each participant who agrees to participate to have their information collected, analysed and stored. Findings will be reported to the Ministry of Health and participating health centres to inform policy and practice as well as disseminated by peer-review publications.

\section{INTRODUCTION}

An increasing number of low and middleincome countries (LMICs) are transitioning

\section{Strengths and limitations of this study}

- Conducting this epidemiological study with sentinel sites facilitates efficient data collection for active surveillance.

- The study is designed to assess multiple outcomes of interest, including follow-up of potential safety signals.

- The sample size is sufficiently large enough to identify adverse events occurring with an incidence of $1: 1000$.

- A limitation is that there is no internal comparison group because patients not using the monitored medicine are not included in the study.

- There are inherent limitations in generalising data collected from sentinel sites to the national level.

to dolutegravir (DTG)-based first-line antiretroviral therapy (ART) regimens following guidelines issued by WHO. ${ }^{12}$ This regimen with two nucleoside reverse transcriptase inhibitors paired with DTG, an integrase inhibitor, is reported to be more effective, with higher viral suppression and CD4 cell count recovery rates and lower risk of treatment discontinuation compared with efavirenz (EFV)-based regimens among treatment-naive adults. $^{2}$ DTG has other advantages over EFV, including a lower potential for drug-drug interactions, more rapid viral suppression and a higher genetic barrier to developing antiretroviral (ARV) drug resistance. DTG is also active against HIV-2 infection, which is naturally resistant to $\mathrm{EFV}^{3}{ }^{3}$ DTG is available in the generic fixeddose formulation at a price comparable with current regimens in most LMICs, making it a favourable option for initiating ART.

A potential safety signal was identified in the Botswana Tsepamo surveillance study. ${ }^{2}$ 
Preliminary results from the Tsepamo study reported an increase in the prevalence of neural tube defects (NTD) among infants born to women who became pregnant while taking DTG-based therapy preconception. There was no evidence of NTD in infants born to a woman who commenced DTG during pregnancy. ${ }^{5}{ }^{6}$ Additional surveillance of birth outcomes in the Tsepamo Study reported a small but significantly higher NTD prevalence among infants exposed to DTG at conception than among women with exposure to non-DTG ART. ${ }^{7}$ Prospectively collected data from the Antiretroviral Pregnancy Registry and European Pregnancy and Paediatric HIV Cohort Collaboration (EPPICC) showed no increased risk for NTDs or other adverse pregnancy or neonatal outcomes following prenatal use of DTG, although sample sizes were small. ${ }^{9}$ Soon thereafter, WHO recommended that women of childbearing age could receive DTG, if they had access to consistent and reliable contraception and highlighted the uncertain evidence regarding the safety of DTG. Since then, however, WHO has recommended DTG-based ART as the preferred first-line regimen for all adults and adolescents with HIV. ${ }^{8}$ Other potential adverse events (AEs) reportedly associated with DTG include increased risk of insomnia and neuropsychiatric adverse reactions. ${ }^{10}$ Available evidence has not shown an increase in the occurrence of other serious AEs such as cardiovascular events, immune reconstitution inflammatory syndrome or suicidal behaviour, in the short term when compared with other ARVs. ${ }^{6}$ Havlir and Doherty called for more data stating that 'large-scale pharmacosurveillance studies of birth outcomes in more countries are the only way to get an answer to the actual risk of neural-tube defects and other adverse birth outcomes'. ${ }^{11}$

The National Directorate of Pharmacy (DNF) and the Mozambican National HIV/AIDS Control Program, with support from the United States Agency for International Development (USAID) Medicines, Technologies and Pharmaceutical Services (MTaPS) program, established an active pharmacovigilance system to monitor AEs and adverse drug reactions (ADRs) in patients, including women of childbearing age, receiving a TLD ART regimen. The specific objectives of this active surveillance activity are to

- Characterise the AE and ADR profile among patients using TLD, including possible interactions between TLD and other medicines.

- Estimate the incidence of AEs, including NTDs and other adverse pregnancy outcomes, in patients using TLD for the treatment of HIV infection, including patients coinfected with TB or MDR-TB and pregnant women.

- Assess causality between observed AEs and the use of TLD for the treatment of HIV infection to determine ADRs.

- Identify risk factors for the development of AEs and ADRs and provide evidence for possible interventions to prevent them.

\section{METHODS}

\section{Study design and eligibility criteria}

This is a prospective, non-interventional, observational cohort study of persons living with HIV who are newly placed on a TLD regimen at sentinel sites. The cohort consists of patients with a diagnosis of HIV, regardless of age and gender commencing treatment with TLD, either as treatment naïve patients or switched from another ARV regimen. Excluded from the cohort are those already taking or switched to TLD prior to commencement of active monitoring, patients who do not consent to be part of the active monitoring or those who are unable to provide a useful medical history for any reason. Patient enrolment commenced in April 2020.

\section{Sample}

Healthcare providers (HCPs) at 10 sentinel sites are requested to enrol eligible patients into the cohort during treatment initiation visits. The intended size of the cohort is 3000 patients to be followed up during routine care visits over 12 months. This sample size provides a 95\% chance of identifying AEs having an incidence of $1: 1000 .^{12}$

\section{Outcomes}

The presence or absence of AEs, as well as additional patient information, is recorded onto a pretested active surveillance data collection form (see online supplemental file annex 1). An AE is defined as 'any untoward medical occurrence that may present during treatment with a pharmaceutical product but which does not necessarily have a causal relationship with this treatment'. ${ }^{13}$ HCPs are requested to monitor for and record the presence or absence of all AEs experienced by the patient during routine clinical care, including AEs of special interest (box 1). All pregnant women, including those who become pregnant during treatment with TLD, are monitored for pregnancy outcomes of interest and other AEs in accordance with the guidance for active monitoring of

\section{Box 1 Outcomes monitored through the active} surveillance activity

$>$ Insomnia.

- Neuropsychiatric events.

- Cardiovascular events.

- Immune reconstitution inflammatory syndrome.

- Stillbirths.

- Miscarriages.

- Small for gestational age.

- Major and minor congenital abnormalities in newborns, including neural tube defects.

- Preterm delivery.

Low birth weight.

- Perinatal death.

- Abnormal laboratory results.

Any other adverse event.

No adverse event. 
pregnant women on TLD (see online supplemental file annex 2). All events and medical conditions are coded using the Medical Dictionary for Regulatory Activities during data entry. ${ }^{14}$

Day ' 0 ' is the day the patient is started on a TLD regimen and enrolled in the cohort for active monitoring. Each enrolled patient will be followed up for up to 12 months. Follow-up visits to monitor and document data on possible ADRs/AEs will be on a monthly basis for the first 3 months after commencement of HIV treatment with TLD per routine patient visit schedule. Subsequent follow-up visits for $\mathrm{ADR} / \mathrm{AE}$ monitoring will be conducted every 3 months. Thus, each patient should have a minimum of six $\mathrm{ADR} / \mathrm{AE}$ monitoring visits throughout the duration of the active surveillance programme. Patients are advised to return to the clinic even before their scheduled follow-up visit if they experience an AE. Alternatively, patients who are able to read and write may be given a paper copy of the follow-up questionnaire to write down AEs that they may have experienced before their next scheduled visit and instructed to bring those forms to their next visit. The National HIV/AIDS control program modified the routine visit schedule for the majority of stable patients on treatment during the COVID-19 lockdown period to quarterly visit. The follow-up schedule for monitored patients was modified accordingly.

Women who become pregnant while on treatment with TLD or before beginning the TLD regimen will be monitored at each antenatal visit up to delivery and the mother and newborn up to 3 months postdelivery. Guidance on active monitoring for pregnant women on TLD (see online supplemental file annex 2) is based, in part, on the country's experience with the Assessment of the Safety of Antimalarial Drug Use During Early Pregnancy study. ${ }^{15}$

A team of HCPs consisting of at least one designated clinician, a nurse and a pharmacist or pharmacy technician at each site was trained on standard operating procedures. Various methods are used to minimise loss to follow-up, such as follow-up phone calls or home visits to collect the necessary data from patients living in distant places from the sentinel site.

\section{Study setting}

The DNF is coordinating the operational implementation of the programme including the establishment and coordination of a national multistakeholder committee to guide and advise overall implementation. The multistakeholder committee consists of representatives from the DNF, HIV programme, TB programme, maternal and child health programme, and other stakeholders as determined by the DNF.

The DNF in collaboration with the national HIV programme selected 10 facilities (table 1) to serve as sentinel sites based on the following criteria:

1. The facility manages at least $200 \mathrm{HIV} / \mathrm{TB}$ coinfected patients monthly.

2. The facility is a referral site that sees at least $100 \mathrm{HIV} /$ TB, including MDR-TB, patients monthly.
Table 1 Sentinel sites included in this active surveillance activity

\begin{tabular}{ll}
\hline Name of site & Province \\
\hline CS Carmelo & Gaza \\
CS Namacurra-Sede & Zambezia \\
CS Macia & Gaza \\
CS Machava II & Maputo Provincia \\
CS Ndlavela & Maputo Provincia \\
HG Polana Caniço & Maputo Cidade \\
CS Mavalane & Maputo Cidade \\
HG Marrere & Nampula \\
CS Cuamba & Niassa \\
HD Gondola & Manica \\
\hline
\end{tabular}

3. Has experience and or ability to follow-up patients during routine follow-up visits (including through phone calls or home visits if necessary).

4. Has capacity for electronic data collection and management (ie, currently has and uses electronic patient management system).

5. Is geographically located to ensure representativeness. Each sentinel site is requested to enrol approximately 300 patients over a period of 6 months.

\section{Patient and public involvement}

Patients and the public were not involved in the design of this study.

\section{Data analysis and management}

The active surveillance monitoring activity uses a combination of electronic and paper-based tools. Forms for initiation and follow-up were based, in part, on generic templates provided by $\mathrm{WHO}$ as well those used for active pharmacovigilance elsewhere in sub-Saharan Africa. ${ }^{916-22}$ The data collection forms are as follows: treatment initiation form (form A), follow-up visit form (form B) and birth outcome and newborn screening form (form C) (see online supplemental file annex 1). Data entry personnel at each site enters the data collected on the paper forms into an electronic data management tool-Pharmacovigilance Monitoring System and transmit the data to the DNF every week. Completed forms are retrieved from the sites during supervisory visits and submitted to the DNF for safekeeping and archiving.

The multistakeholder active safety monitoring committee will use the WHO Uppsala Monitoring Centre causality assessment method to assess the $\mathrm{AE}$ reports to determine the relationship between reported AEs and medicine(s) taken by patients. ${ }^{23}$ The committee will classify AEs into two groups:

Group 1: events determined as having a certain, probable or possible relationship with the monitored medicine. These will be classified as 'adverse drug reactions' because they are likely to be related to the use of the 
monitored medicine and entered into the national ADR database.

Group 2: events determined as having an unlikely or unassessable relationship with medicine use. These will be classified as 'incidentals' as their occurrence is likely to be incidental to the use of the monitored medicine. These will be maintained in the active monitoring database.

The DNF will add AEs assessed as having a certain, probable or possible relationship with the use of TLD to the national database of ADRs that are received through the spontaneous reporting system. All reported AEs that are assessed as having an unlikely relationship with the use of TLD will be left in the database of reports from the active monitoring system. The DNF will periodically analyse the data (monthly at the initial stage and quarterly thereafter) and generate descriptive frequency tables for demographics, medicine use and adverse medicine events.

A mid-term (approximately 6-8 months after commencement of active monitoring) interim analysis and the main analysis at the conclusion of the follow-up period will be performed to determine adverse reaction profiles. The incidence of an ADR and AE will be calculated as the number of events divided by the total number of patient-months of follow-up. These estimates will be determined with 95\% CIs assuming a Poisson distribution. Stratification and adjustments will be made as appropriate using collected baseline information, including comedication and comorbidities as collected through the data collection forms. Frequencies of ADRs will be compared by medicine category using univariate analysis and expressed as relative risks with corresponding $95 \%$ CIs and population attributable risk by ART and other medicines.

HCPs, data entry personnel and data analysts involved in the process were trained on how to use the data collection tools and other aspects of active monitoring. Initial support supervision was conducted at each facility via phone calls within the first 2 months of patient enrolment. Further routine support supervision is provided to the sites monthly via phone calls due to COVID-19 restrictions. Quarterly in-person visits will commence in 2021. The DNF reviews the reports collected regularly for data quality and provide the necessary feedback for improving the quality of collected data.

\section{Ethics and dissemination}

Written informed consent is obtained from each participant who consents to participate and have his or her information collected, analysed and stored. In addition, patients are informed that their participation in the programme is voluntary and they have the right to withdraw at any time and continue to receive care at the facility. Those who are between the ages of 12 and 18 years are required to sign an assent form to participate in the programme. In the event of children who are more than $30 \mathrm{~kg}$ and less than 12 years old, informed written consent is obtained from their parents.
Confidentiality of patient data is maintained by limiting access to the information contained in the paper and electronic tools to only persons involved in the active monitoring of patients and who need to access the data for purposes of data entry, review or analysis. The DNF will enforce appropriate data access control measures and deidentify all data before sharing. Data from this active safety monitoring study will be stored by the DNF for 5 years after the programme ends.

Findings from the TLD active monitoring activity will be disseminated to relevant stakeholders through quarterly newsletters or reports. The DNF, in collaboration with the multistakeholder active monitoring committee and with support from MTaPS, will develop, review and disseminate the newsletter or report. The DNF will use information leaflets to inform HCPs and patients of the recommendations derived from the findings. Findings and recommendations from the activity will also be shared with a wider audience through the publication of scientific and professional articles in peer-reviewed journals following due process.

The 2019 guidelines from WHO provide reassurance of DTG as the preferred ARV drug in first and secondline regimens due to the declining estimate of NTD risk and observed efficacy. ${ }^{24}$ Major factors that can influence the transition to DTG include active surveillance of emerging toxicity issues and systems for monitoring safety in pregnancy.

\section{Author affiliations}

${ }^{1}$ Pharmacovigilance Unit, National Directorate of Pharmacy, Maputo, Mozambique ${ }^{2}$ National HIV/AIDS Division, MISAU, Maputo, Mozambique

${ }^{3}$ MTaPS, Management Sciences for Health, FCT, Nigeria

${ }^{4}$ Medicine, Technologies and Pharmaceutical Services (MTaPS), Management Sciences for Health, Maputo, Mozambique

${ }^{5}$ Medicines, Technologies and Pharmaceutical Sciences (MTaPS), Management Sciences for Health Inc Arlington, Arlington, Virginia, USA

${ }^{6}$ Department of Pharmacy, University of Washington School of Public Health, Seattle, Washington, USA

${ }^{7}$ School of Pharmacy, University of Washington Seattle Campus, Seattle, Washington, USA

\section{Twitter Comfort Kunak Ogar @ComfortKOGAR}

Contributors CKO conceived the idea for the publication of the manuscript and drafted the protocol with support from MM, IG, LN, SV, EDS, DN and AS. TVS and $\mathrm{AC}$ reviewed and approved the implementation of the protocol. TH drafted the manuscript. JMP, AL and all authors reviewed and approved the manuscript.

Funding This work was supported by the US Agency for International Development (USAID) under contract number (7200AA18C00074). The contents are the responsibility of the authors and do not necessarily reflect the views of USAID or the US Government.

Competing interests None declared.

Patient consent for publication Not required.

Provenance and peer review Not commissioned; externally peer reviewed.

Supplemental material This content has been supplied by the author(s). It has not been vetted by BMJ Publishing Group Limited (BMJ) and may not have been peer-reviewed. Any opinions or recommendations discussed are solely those of the author(s) and are not endorsed by BMJ. BMJ disclaims all liability and responsibility arising from any reliance placed on the content. Where the content includes any translated material, BMJ does not warrant the accuracy and reliability of the translations (including but not limited to local regulations, clinical guidelines, 
terminology, drug names and drug dosages), and is not responsible for any error and/or omissions arising from translation and adaptation or otherwise.

Open access This is an open access article distributed in accordance with the Creative Commons Attribution Non Commercial (CC BY-NC 4.0) license, which permits others to distribute, remix, adapt, build upon this work non-commercially, and license their derivative works on different terms, provided the original work is properly cited, appropriate credit is given, any changes made indicated, and the use is non-commercial. See: http://creativecommons.org/licenses/by-nc/4.0/.

ORCID iD

Comfort Kunak Ogar http://orcid.org/0000-0003-0448-7111

\section{REFERENCES}

1 WHO. Consolidated guidelines on the use of antiretroviral drugs for treating and preventing HIV infection. Recommendations for a public health approach - Second edition. 2nd ed. Geneva: World Health Organization, 2018. http://www.who.int/hiv/pub/arv/arv-2016/en/

2 WHO. Updated recommendations on first-line and secondline antiretroviral regimens and post-exposure prophylaxis and recommendations on early infant diagnosis of HIV: interim guidelines: supplement to the 2016 consolidated guidelines on the use of antiretroviral drugs for treating and preventing HIV infection. Geneva: WHO, 2018. https://apps.who.int/iris/bitstream/handle/10665/ 277395/WHO-CDS-HIV-18.51-eng.pdf

3 Pujari S, Patel A, Gaikwad S, et al. Effectiveness of dolutegravirbased antiretroviral treatment for HIV-2 infection: retrospective observational study from Western India. J Antimicrob Chemother 2020;75:1950-4.

4 Treviño A, Cabezas T, Lozano AB, et al. Dolutegravir for the treatment of HIV-2 infection. J Clin Virol 2015;64:12-15.

5 WHO. Potential safety issue affecting women living with HIV using dolutegravir at the time of conception. Geneva: World Health Organization, 2018.

6 Dorward J, Lessells R, Drain PK, et al. Dolutegravir for first-line antiretroviral therapy in low-income and middle-income countries: uncertainties and opportunities for implementation and research. Lancet HIV 2018;5:e400-4.

7 Zash R, Holmes L, Diseko M, et al. Neural-Tube defects and antiretroviral treatment regimens in Botswana. N Engl J Med 2019;381:827-40.

8 Raesima MM, Ogbuabo CM, Thomas V, et al. Dolutegravir use at conception - additional surveillance data from Botswana. N Engl J Med Overseas Ed 2019;381:885-7.
9 Vannappagari V, Thorne C, for APR and EPPICC. Pregnancy and neonatal outcomes following prenatal exposure to dolutegravir. $J$ Acquir Immune Defic Syndr 2019;81:371-8.

10 Lepik KJ, Yip B, Ulloa AC, et al. Adverse drug reactions to integrase strand transfer inhibitors. AIDS 2018;32:903-12.

11 v HD, Doherty MC. Global HIV treatment - turning Headwinds to Tailwinds. New England Journal of Medicine 2019;381:873-4.

12 Strom BL, Kimmel SE, Hennessy S, eds. Pharmacoepidemiology. 6th ed. Hoboken: Wiley-Blackwell, 2019.

13 Tinto H, Sevene E, Dellicour S, et al. Assessment of the safety of antimalarial drug use during early pregnancy (ASAP): protocol for a multicenter prospective cohort study in Burkina Faso, Kenya and Mozambique. Reprod Health 2015;12:112.

14 MedDRA. Medical dictionary for regulatory activities, March 2020. Available: https://www.meddra.org/ [Accessed December 2, 2020].

15 WHO-UMC. The use of the WHO-UMC system for standardised case causality assessment why causality assessment? 2020. Available: https://www.who.int/medicines/areas/quality_safety/safety_efficacy/ WHOcausality_assessment.pdf

16 WHO. WHO implementation tool for monitoring the toxicity of new antiretroviral and antiviral medicines in HIV and viral hepatitis programmes. Geneva: World Health Organization, 2018. http://www. who.int/hiv/pub/arv toxicity/arv-toxicity-monitoring-tool/en/

17 Bassi PU, Osakwe Al, Isah A, et al. Safety of artemisinin-based combination therapies in Nigeria: a cohort event monitoring study. Drug Saf 2013;36:747-56.

18 Suku CK, Hill G, Sabblah G, et al. Experiences and lessons from implementing cohort event monitoring programmes for antimalarials in four African countries: results of a questionnaire-based survey. Drug Saf 2015;38:1115-26.

19 Dodoo ANO, Fogg C, Nartey ET, et al. Profile of adverse events in patients receiving treatment for malaria in urban Ghana: a cohortevent monitoring study. Drug Saf 2014;37:433-48.

20 Mann M, Mengistu A, Gaeseb J, et al. Sentinel site active surveillance of safety of first-line antiretroviral medicines in Namibia. Pharmacoepidemiol Drug Saf 2016;25:1052-60.

21 Mann M, Mengistu A, Gaeseb J, et al. Active surveillance versus spontaneous reporting for first-line antiretroviral medicines in Namibia: a cost-utility analysis. Drug Saf 2016;39:859-72.

22 Corbell C, Katjitae I, Mengistu A, et al. Records linkage of electronic databases for the assessment of adverse effects of antiretroviral therapy in sub-Saharan Africa. Pharmacoepidemiol Drug Saf 2012;21:407-14.

23 European database of suspected adverse drug reaction reports. Glossary, 2020. Available: http://www.adrreports.eu/en/glossary.html

$24 \mathrm{WHO}$. Update of recommendations on first-and second-line antiretroviral regimens. Geneva: World Health Organization, 2019. https://apps.who.int/iris/bitstream/handle/10665/325892/WHO-CDSHIV-19.15-eng.pdf?ua=1 